\title{
Multiple solutions in extracting physics information from experimental data
}

\author{
C. Z. Yuan, X. H. Mo†, P. Wang ${ }^{\ddagger}$ \\ Institute of High Energy Physics, Chinese Academy of Sciences, Beijing 100049, China
}

\begin{abstract}
Multiple solutions exist in various experimental situations whenever the sum of several amplitudes is used to fit the experimentally measured distributions, such as the cross section, the mass spectrum, or the angular distribution. We show a few examples where multiple solutions were found, while only one solution was reported in the publications. Since there is no existing rules found in choosing any one of these solutions as the physics one, we propose a simple rule which agrees with what have been adopted in previous literatures: the solution corresponding to the minimal magnitudes of the amplitudes must be the physical solution. We suggest test this rule in the future experiments.
\end{abstract}

Key words Amplitude, Multi-solution, mixing, physics solution

\section{Introduction}

In quantum mechanics, a physics observable is proportional to the modulus of the amplitude squared. In case of more than one amplitude contributing to a process, they are summed to obtain the total amplitude (generally there are relative phases between these amplitudes), and thus one generally has contribution from interference term to the physics observable. It is simple to predict a physics observable when the amplitudes and the relative phases between them are known, since there is no ambiguity in this procedure.

However, in many circumstances, the experimental quantities are measured, and from which we extract the information on the amplitudes. As there is a square operation between the observable and the amplitudes, we would expect multiple solutions in solving the equation from a pure mathematics point of view. Then we face a common problem: which solution is the physics one.

In this Letter, we present a few examples where two-solutions were reported in fitting with the coherent sum of two amplitudes; and revisit a few cases where only one solution

*yuancz@ihep.ac.cn

${ }^{\dagger}$ moxh@ihep.ac.cn

${ }^{\ddagger}$ wangp@ihep.ac.cn 
was reported and we find the other distinctive solution in fitting with two amplitudes; we also discuss more complicated situations where more than two amplitudes are used to fit the data. Finally, we propose a conjecture on how to choose from multiple solutions the physics one.

\section{Examples with two-solutions reported}

A few recent examples reporting multiple solutions are in the study of the so-called $Y$ states via initial state radiation $(I S R)$ by the Belle experiment. Figure 1 shows the invariant mass distributions of $\pi^{+} \pi^{-} J / \psi$ and $\pi^{+} \pi^{-} \psi(2 S)$ after all the selection in Belle data [1, 2], together with a fit with two coherent resonant terms and an incoherent background term. Table 1 shows the fit results, including the $Y(4008)$ and $Y(4260)$ from the $\pi^{+} \pi^{-} J / \psi$ mode, and the $Y(4360)$ and $Y(4660)$ from the $\pi^{+} \pi^{-} \psi(2 S)$ mode. It should be noted that in both channels there are two solutions of exactly the same goodness-of-the-fit, with exactly the same mass and width for the resonances but with very different coupling to $e^{+} e^{-}$pair $\left(\Gamma_{e^{+} e^{-}}\right)$. Instead of choosing one from the two solutions, Belle reported both in their publications, however, Particle Data Group (PDG) quoted sometimes only one of the solutions in averaging with those from other experiments [3]. Such a treatment of data is rather suspicious, since the solutions picked up randomly from different experiment may have distinctive features and should not be averaged together.

Another example is the study of the decay dynamics of $\eta^{\prime} \rightarrow \gamma \pi^{+} \pi^{-}$mode. When the $\pi^{+} \pi^{-}$invariant mass distribution is fitted with coherent sum of the $\rho$ resonance and a contact term, it is found that there are two solutions of equal goodness-of-the-fit. One solution corresponds to constructive interference between the two amplitudes while the other destructive interference [4]. Similar study in previous analyses only reported one solution [5] which corresponds to the solution with constructive interference in the BES data [4].

\section{Examples with one-solution reported}

As the examples shown in the previous section are basically a fit to an $s$-dependent distribution with two coherent amplitudes, one would expect that there would be in general two solutions in such a circumstance. In the literatures, there are many such kinds of fits, especially in the low energy $e^{+} e^{-}$annihilation experiments, where the cross section of $e^{+} e^{-} \rightarrow$ hadrons is parameterized as the coherent sum of the amplitudes of the vector mesons. We show two typical examples below where only one solution was reported, and we redo the fit to obtain the other solution from the data. 

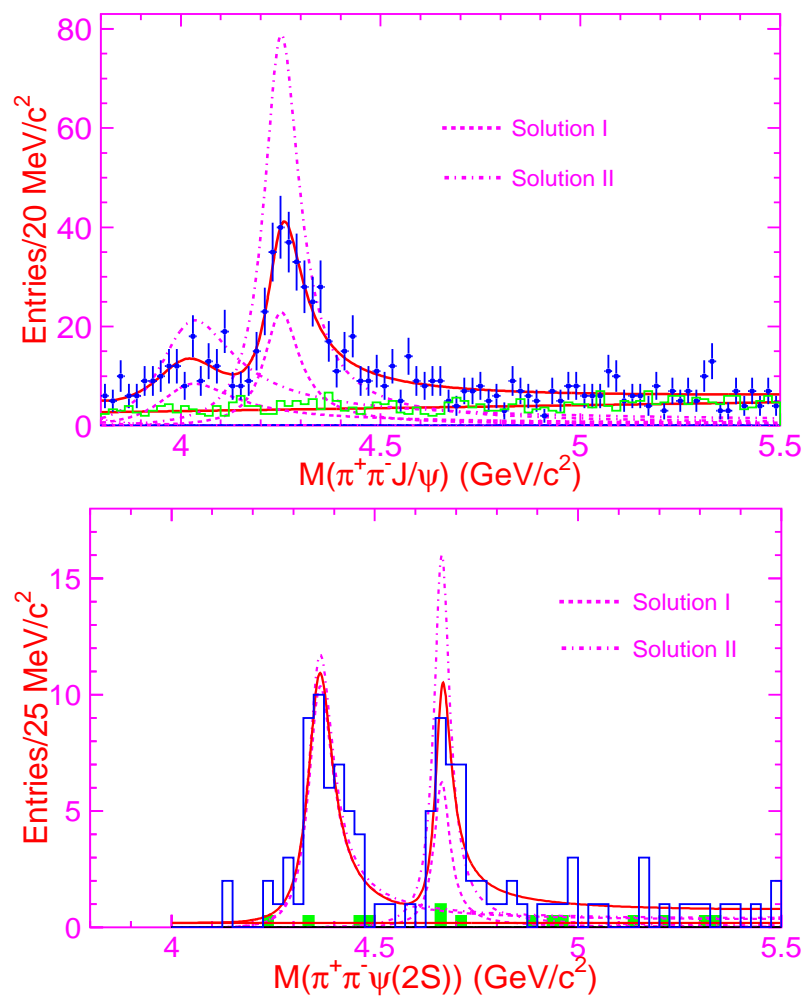

Figure 1: The $\pi^{+} \pi^{-} J / \psi$ (upper) and $\pi^{+} \pi^{-} \psi(2 S)$ (lower) invariant mass distributions and the best fit with two coherent resonances together with a background term. The data are from Belle [1, 2].

\subsection{Branching fraction of $\phi \rightarrow \omega \pi^{0}$}

The most precise data on $e^{+} e^{-} \rightarrow \omega \pi^{0}$ near the $\phi$ resonance were reported by KLOE [6] with both $\omega \rightarrow \pi^{+} \pi^{-} \pi^{0}$ and $\omega \rightarrow \gamma \pi^{0}$. The cross section as a function of $\sqrt{s}$ is parameterized as

$$
\sigma(\sqrt{s})=\sigma_{n r}(\sqrt{s}) \cdot\left|1-Z \frac{M_{\phi} \Gamma_{\phi}}{D_{\phi}(\sqrt{s})}\right|^{2}
$$

in the KLOE paper [6], where $\sigma_{n r}(\sqrt{s})=\sigma_{0}+\sigma^{\prime}\left(\sqrt{s}-M_{\phi}\right)$ is the bare cross section for the non-resonant process, parameterized as a linear function of $\sqrt{s} ; Z$ is the interference parameter, while $M_{\phi}, \Gamma_{\phi}$ and $D_{\phi}=M_{\phi}^{2}-s-i M_{\phi} \Gamma_{\phi}$ are the mass, the width, and the inverse propagator of the $\phi$ meson, respectively.

We take the KLOE data from Table I of Ref. [6] with $\omega \rightarrow \pi^{+} \pi^{-} \pi^{0}$ and fit with the same parametrization given above. In our fit, the Born-order cross section is calculated as $\sigma_{\text {vis }} / \delta_{\text {rad }}$, and only statistical errors are considered in the $\chi^{2}$ construction. Table 2 shows the results from our fit, where two solutions are found with the same fit quality. We can see that the parameters from Solution I are quite similar to those listed in Table II of Ref. [6], the slight difference is due to the simplified procedure for the Born-order cross section calculation in our fit. It is found that Solution II has a much larger resonant amplitude, 
Table 1: Fit results of the $\pi^{+} \pi^{-} J / \psi$ and $\pi^{+} \pi^{-} \psi(2 S)$ invariant mass spectra. The first errors are statistical and the second systematic. $M, \Gamma_{\text {tot }}$, and $\mathcal{B} \cdot \Gamma_{e^{+} e^{-}}$are the mass (in $\mathrm{MeV}$ ), total width (in $\mathrm{MeV}$ ), product of the branching fraction to hadronic mode and the $e^{+} e^{-}$partial width (in $\mathrm{eV}$ ), respectively. $\phi$ is the relative phase between the two resonances (in degrees).

\begin{tabular}{ccc}
\hline \hline Parameters & Solution I & Solution II \\
\hline$M(Y(4008))$ & \multicolumn{2}{c}{$4008 \pm 40_{-28}^{+114}$} \\
$\Gamma_{\text {tot }}(Y(4008))$ & \multicolumn{2}{c}{$226 \pm 44 \pm 87$} \\
$\mathcal{B} \cdot \Gamma_{e^{+} e^{-}}(Y(4008))$ & $5.0 \pm 1.4_{-0.9}^{+6.1}$ & $12.4 \pm 2.4_{-1.1}^{+14.8}$ \\
$M(Y(4260))$ & \multicolumn{2}{c}{$4247 \pm 12_{-32}^{+17}$} \\
$\Gamma_{\text {tot }}(Y(4260))$ & \multicolumn{2}{c}{$108 \pm 19 \pm 10$} \\
$\mathcal{B} \cdot \Gamma_{e^{+} e^{-}}(Y(4260))$ & $6.0 \pm 1.2_{-0.5}^{+4.7}$ & $20.6 \pm 2.3_{-1.7}^{+9.1}$ \\
$\phi$ & $12 \pm 29_{-98}^{+7}$ & $-111 \pm 7_{-31}^{+28}$ \\
\hline$M(Y(4360))$ & \multicolumn{2}{c}{$4361 \pm 9 \pm 9$} \\
$\Gamma_{\text {tot }}(Y(4360))$ & \multicolumn{2}{c}{$74 \pm 15 \pm 10$} \\
$\mathcal{B} \cdot \Gamma_{e^{+} e^{-}}(Y(4360))$ & $10.4 \pm 1.7 \pm 1.5$ & $11.8 \pm 1.8 \pm 1.4$ \\
$M(Y(4660))$ & \multicolumn{2}{c}{$4664 \pm 11 \pm 5$} \\
$\Gamma_{\text {tot }}(Y(4660))$ & \multicolumn{2}{c}{$48 \pm 15 \pm 3$} \\
$\mathcal{B} \cdot \Gamma_{e^{+} e^{-}}(Y(4660))$ & $3.0 \pm 0.9 \pm 0.3$ & $7.6 \pm 1.8 \pm 0.8$ \\
$\phi$ & $39 \pm 30 \pm 22$ & $-79 \pm 17 \pm 20$ \\
\hline \hline
\end{tabular}

and the resulting branching fraction of the isospin violating process $\phi \rightarrow \omega \pi^{0}$ is at per mille level, about two orders of magnitude higher than Solution I, namely, the solution reported in the original work [6]. Figure 2 shows the fit results and the contribution of each component in the fit. Similarly, we try a fit to the cross sections measured with $\omega \rightarrow \gamma \pi^{0}$, there are also two solutions obtained from the fit and the results are in good agreement with those from $\omega \rightarrow \pi^{+} \pi^{-} \pi^{0}$.

\section{$3.2 \quad F_{\pi}$ and the $\rho-\omega$ mixing}

Even before the discovery of the $\tau$ lepton, Tsai calculated the branching fractions of such a heavy lepton decaying into vector final states by the conserved vector current (CVC) hypothesis using $e^{+} e^{-} \rightarrow \pi^{+} \pi^{-}$data measured in $e^{+} e^{-}$annihilation experiments [7]. This calculation, referred to as $\mathcal{B}_{\tau^{-} \rightarrow \pi^{-} \pi^{0} \nu_{\tau}}^{\mathrm{CVC}}$, was tested ever since the discovery of the $\tau$ lepton. As the precisions of both $e^{+} e^{-}$annihilation and $\tau$ decay experiments improved significantly 
Table 2: Results from fits to the $e^{+} e^{-} \rightarrow \omega \pi^{0}$ cross sections measured with $\omega \rightarrow \pi^{+} \pi^{-} \pi^{0}$.

\begin{tabular}{ccc}
\hline \hline Parameter & Solution I & Solution II \\
\hline$\sigma_{0}[\mathrm{nb}]$ & $7.88 \pm 0.04$ & $7.88 \pm 0.08$ \\
$\Re(Z)$ & $0.106 \pm 0.004$ & $0.106 \pm 0.006$ \\
$\Im(Z)$ & $-0.103 \pm 0.003$ & $-1.90 \pm 0.006$ \\
$\sigma^{\prime}[\mathrm{nb} / \mathrm{MeV}]$ & $0.064 \pm 0.002$ & $0.064 \pm 0.006$ \\
$\mathcal{B}\left(\phi \rightarrow \omega \pi^{0}\right)$ & $4.61 \times 10^{-5}$ & $7.62 \times 10^{-3}$ \\
\hline \hline
\end{tabular}

in the past three decades, the test has reached the precision of better than $1 \%$ level, and the discrepancy of $2 \sigma$ level between $\mathcal{B}_{\tau^{-} \rightarrow \pi^{-} \pi^{0} \nu_{\tau}}^{\mathrm{CVC}}$ and $\mathcal{B}_{\tau^{-} \rightarrow \pi^{-} \pi^{0} \nu_{\tau}}$ is observed [8]. Many theoretical efforts have been made to understand this discrepancy, including a better understanding of the isospin violation correction and so on.

One source of the isospin breaking correction is the $\rho$ - $\omega$ mixing effect in the $e^{+} e^{-}$ annihilation data which is absent in the $\tau$ decays. The mixing effect on $\mathcal{B}_{\tau^{-} \rightarrow \pi^{-} \pi^{0} \nu_{\tau}}^{\mathrm{CVC}}$ is estimated by fitting the $e^{+} e^{-} \rightarrow \pi^{+} \pi^{-}$data in the vector meson dominance (VMD) model (including $\rho$ and its excited states and $\omega$ ) and subtract the $\omega$ contribution by setting its amplitude to zero. This has been carried out in many previous analyses [8, 9, 10], but in all cases only one solution was reported. As a heuristic example, we only use the CMD2 data and follow the fit described in the CMD2 paper [11], namely, with the GSparametrization [12] of the $F_{\pi}$ :

$$
F_{\pi}^{\mathrm{GS}}(s)=\frac{\mathrm{BW}_{\rho}^{\mathrm{GS}}(s)\left[1+\delta \frac{s}{m_{\omega}^{2}} P_{\omega}(s)\right]+\beta \mathrm{BW}_{\rho^{\prime}}^{\mathrm{GS}}(s)}{1+\beta},
$$

where

$$
\begin{aligned}
\mathrm{BW}_{V}^{\mathrm{GS}}(s) & =\frac{m_{V}^{2}\left(1+d \cdot \Gamma_{V} / m_{V}\right)}{m_{V}^{2}-s+f(s)-i m_{V} \Gamma_{V}(s)}, \\
P_{\omega}(s) & =\frac{m_{\omega}^{2}}{m_{\omega}^{2}-s-i m_{\omega} \Gamma_{\omega}} .
\end{aligned}
$$

The definitions of all the quantities can be found in Refs. [10, 12]. Here, complex numbers $\delta$ and $\beta$, as well as the mass and width of the $\rho$, are fit parameters. The masses and widthes of $\omega$ and $\rho^{\prime}$ are fixed to their PDG values [3], and the phase of $\beta$ is fixed to $180^{\circ}$.

We found two solutions in our fit, as shown in Fig. 3 and Table 3, It is clear that Solution I is in good agreement with the results reported by CMD2 experiment [11], and the resulting $\rho-\omega$ mixing corrections to $\mathcal{B}_{\tau^{-} \rightarrow \pi^{-} \pi^{0} \nu_{\tau}}^{\mathrm{CVC}}$ and $a_{\mu}$ are in agreement with the results in Refs. [8, 9]. However, the relative strength between $\omega$ and $\rho$ is different in the 

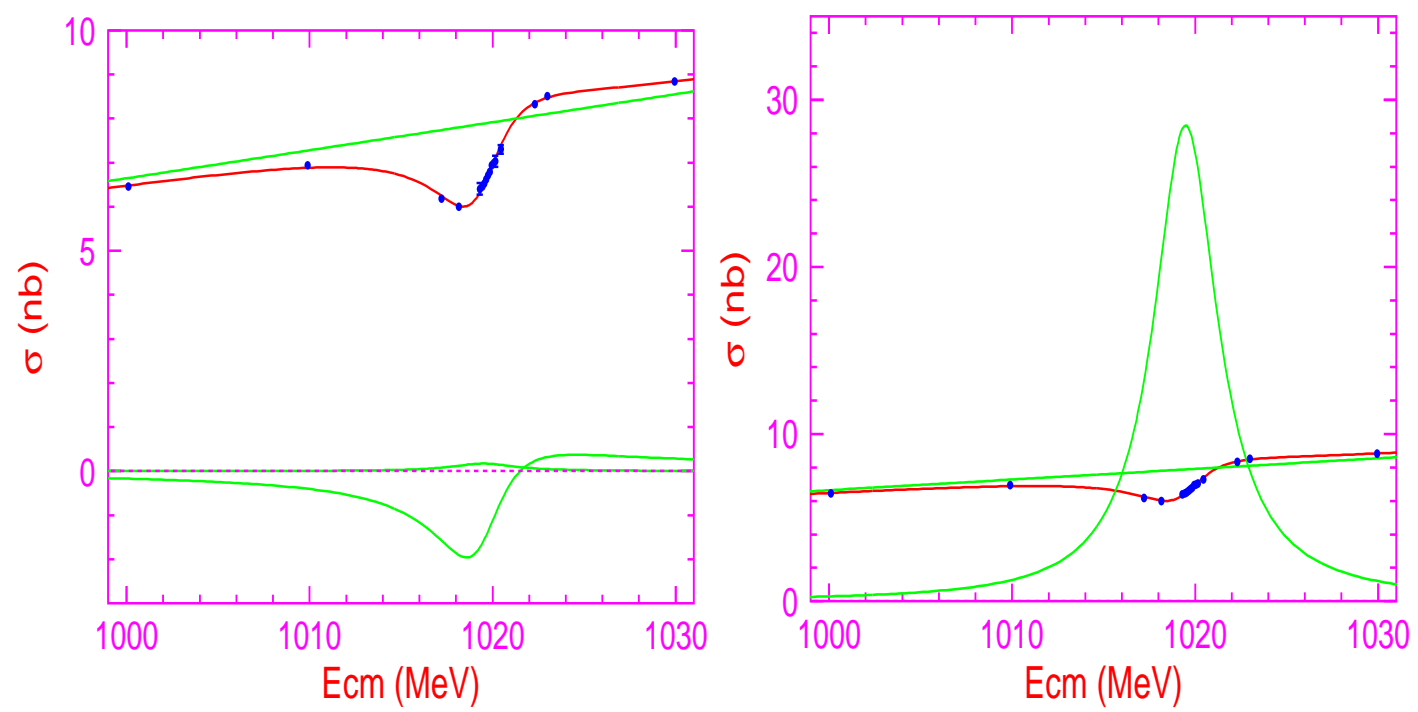

Figure 2: Fit to the $e^{+} e^{-} \rightarrow \omega \pi^{0}$ cross sections as a function of center-of-mass energy. Data points are shown with dots with error bars. Top plot is for solution I: at $\sqrt{s}=$ $1.019 \mathrm{GeV}$, from top to bottom, the solid curves are continuum, best fit, resonance, and interference terms. Bottom plot is for Solution II: at $\sqrt{s}=1.019 \mathrm{GeV}$, from top to bottom, the solid curves are resonance, continuum, and best fit, the interference term is not shown.

other solution, and the corrections to $\mathcal{B}_{\tau^{-} \rightarrow \pi^{-} \pi^{0} \nu_{\tau}}^{\mathrm{CVC}}$ and $a_{\mu}$ are different too, as shown in Table 3 .

As the amplitude of the $\omega$ term in Solution II is more than an order of magnitude higher than that in Solution I, we would expect a branching fraction of $\omega \rightarrow \pi^{+} \pi^{-}$much larger than that reported in previous analyses: about $70 \%$ in Solution II versus $1.5 \%$ in Solution I. It seems Solution II is in contradiction with our expectation that the isospin breaking decay $\omega \rightarrow \pi^{+} \pi^{-}$should be much smaller than the isospin conserving decay $\omega \rightarrow \pi^{+} \pi^{-} \pi^{0}$. However, it is obvious that we are used to the small $\omega \rightarrow \pi^{+} \pi^{-}$branching fraction because we have never thought about the possibility of the existence of the second solution in the fit.

\section{Examples with more than two amplitudes}

The examples shown above are described by the sum of two amplitudes with a free relative strength and relative phase. In the case of more than two amplitudes contributing to the distribution, the number of solutions is $2^{n-1}$, where $n$ is the number of free amplitudes.

In the fit to the $e^{+} e^{-} \rightarrow \phi \pi^{+} \pi^{-}$cross sections [13, incoherent and coherent sums of two amplitudes (the $\phi(1680)$ and $Y(2175)$ ) are tested, while in estimating the significance of 

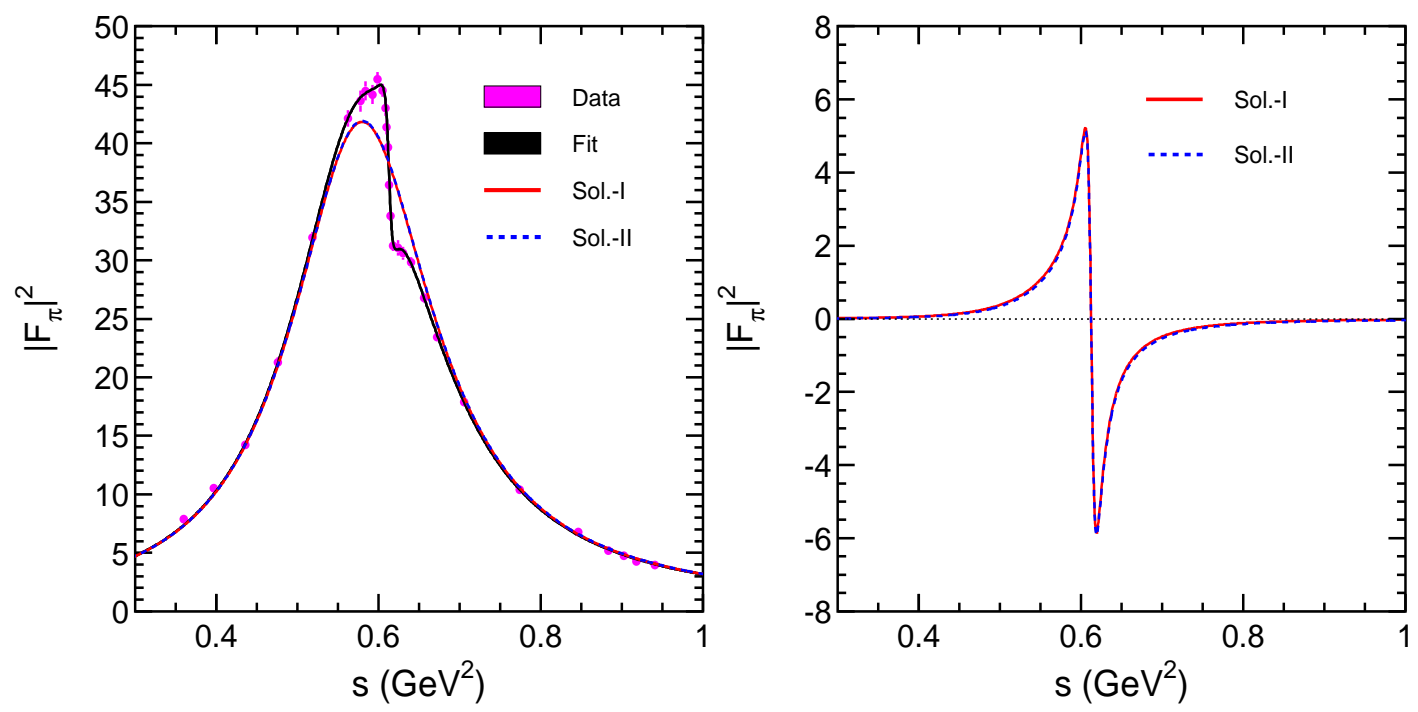

Figure 3: Fit to the $e^{+} e^{-} \rightarrow \pi^{+} \pi^{-}$form factors below $s=1 \mathrm{GeV}^{2}$ measured at CMD2 [10]. Top: along the data points (dots with error bars) is the best fit. The $I=1$ part of the fit is shown for the two solutions. Bottom: comparison of the $\rho-\omega$ interference part in two solutions.

a third resonance $(X(2400))$, Belle also tested the possibility of the coherent sum of three amplitudes. This procedure is repeated with combined BaBar [14] and Belle data [13] in Ref. [15. In this latter case, four solutions are found with the same masses and widthes for the resonances, but with different coupling constants.

The same phenomenon was also shown in the fit to the combined BaBar [16] and Belle [2] data on $e^{+} e^{-} \rightarrow \pi^{+} \pi^{-} \psi(2 S)$ [17], where two pairs of solutions were found when fitting with the coherent sum of the $Y(4360), Y(4660)$, and $Y(4260)$.

Table 3: Results from fits to the $e^{+} e^{-} \rightarrow \pi^{+} \pi^{-}$form factors measured at CMD2 [10].

\begin{tabular}{ccc|c}
\hline \hline Parameter & Solution I & Solution II & Davier [8] \\
\hline$m_{\rho}[\mathrm{MeV}]$ & \multicolumn{2}{c}{$775.9 \pm 0.5$} & - \\
$\Gamma_{\rho}[\mathrm{MeV}]$ & \multicolumn{2}{c}{$146.0 \pm 0.8$} & - \\
$|\delta|\left[\times 10^{-3}\right]$ & $1.62 \pm 0.06$ & $21.97 \pm 0.04$ & - \\
$\phi_{\delta}\left[^{\circ}\right]$ & $10.1 \pm 1.4$ & $86.56 \pm 0.17$ & - \\
$|\beta|$ & $0.086 \pm 0.004$ & - \\
\hline$\Delta \mathcal{B}^{\text {mixing }}[\%]$ & $-0.03 \pm 0.01$ & $+0.04 \pm 0.01$ & $-0.01 \pm 0.01$ \\
$\Delta a_{\mu}^{\text {mixing }}\left[10^{-10}\right]$ & $+2.5 \pm 0.2$ & $+1.6 \pm 0.2$ & $+2.80 \pm 0.19$ \\
\hline \hline
\end{tabular}




\section{Partial wave analysis}

All the examples discussed so far are one-dimensional problems. In the case of fitting a multi-dimensional distribution, the same multi-solution problem also exists. The partial wave analysis (PWA) and the Dalitz plot analysis techniques used widely in hadron physics potentially have such a problem.

In a recent BES analysis of the $\psi(2 S) \rightarrow \pi^{+} \pi^{-} J / \psi$ process [18, the $\pi^{+} \pi^{-}$system is fitted with contributions from $\sigma$, a contact term, and a possible tensor amplitude $f_{2}$. It is curious to us why the two very similar models shown in Figure 4 of Ref. [18] give very different fractions of the $\sigma$ term. The aforementioned expositions lead us to suspect that two different solutions are found in the two models, one with constructive interference and the other with strong destructive interference. The authors may try to excavate the other solutions in each of those models used in fitting the experimental data. Fortunately, according to the study above, we found that all different solutions result in identical line shape of the resonance (the same mass and width), so the resonant parameters should be correct even if the branching fractions obtained from these analyses are not reliable.

A general partial wave analysis may have many amplitudes and all of them are added coherently to fit the data distributions. In principle, there are multiple solutions, although we cannot judge how many fold of ambiguity. In some circumstances, when more than one solutions are found, a simple average is taken to be the best estimation of the parameters, this is obviously non-physical, since any one of the solutions is a good description of the data but the average is generally not; while in some other cases, this is just overlooked, which makes the comparison between experiments looks very strange, since one compares two different solutions from two experiments which are different by definition. Herein, great care must be taken in treating the magnitudes of the amplitudes in extracting them from multi-dimensional distributions with the coherent sum of many amplitudes.

\section{Minimal amplitude conjecture}

In preceding sections, we examined a few cases where amplitudes are extracted from experimentally observed distributions, and in all the cases more than one solutions were found. We also discussed the similar problem in PWA. All these indicate that this problem actually is common in data analysis. Up to now, this problem has not been taken into consideration seriously. Moreover, no one has thought about how to pick up one solution from all the possibilities, and there is indeed lack of solid physics argument on how to choose one of the solutions as the physics one.

However, from the existing examples and our observation, we notice that the solution with the smallest modulus of the amplitude agrees with present expectation, such as in 
the $\phi \rightarrow \omega \pi^{0}$ and the $\rho$ - $\omega$ mixing cases. So it may not be very surprising that the physics solution of any system corresponds to the one where the modula of the amplitudes take the minimal values among all the solutions. We call this selection rule "the minimal amplitude conjecture".

Such a rule can be comprehended readily from a simple "economic" principle. One would expect that a solution in which the physics observable is produced via two or more very large amplitudes with strong destructive interference is less "economic" than the one via small amplitudes but with constructive interference. In the latter case, we always find maximum constructive interference among amplitudes. With this economic "minimal amplitude conjecture", we can determine the unique physics solutions of all the aforementioned examples. This will make the life much simpler.

\section{Conclusion}

In this Letter, we put forth two important problems: multiple solutions in fitting experimental data and the selection of the physics solution among many possible solutions. As a matter of fact, more and more cases with multiple solutions appear in recent data analyses and even more may have been overlooked previously. Here, we strongly suggest that all possible solutions be found out and reported in the future analyses, in order to avoid a babel of arguments and misleading theoretical deductions.

When confronting with multiple solutions, one has to make a choice. We propose a "minimal amplitude conjecture": the solution with minimal modula of the amplitudes is the physics one. Such a rule ensures a unique solution is singled out from multiple solutions. This conjecture could be treated as a principle which should be tested experimentally by the future data analyses.

\section{Acknowledgements}

This work is supported in part by the National Natural Science Foundation of China (10775412, 10825524, 10935008), the Instrument Developing Project of the Chinese Academy of Sciences (YZ200713), Major State Basic Research Development Program (2009CB825203, 2009CB825206), and Knowledge Innovation Project of the Chinese Academy of Sciences (KJCX2-YW-N29).

\section{References}

[1] C. Z. Yuan et al. [Belle Collaboration], Phys. Rev. Lett. 99 (2007) 182004 arXiv:0707.2541 [hep-ex]]. 
[2] X. L. Wang et al. [Belle Collaboration], Phys. Rev. Lett. 99 (2007) 142002 arXiv:0707.3699 [hep-ex]].

[3] C. Amsler et al. [Particle Data Group], Phys. Lett. B 667 (2008) 1, and 2009 partial update for the 2010 edition.

[4] H. X. Chen [BES Collaboration], Int. J. Mod. Phys. A 22 (2007) 637.

[5] M. Benayoun, P. David, L. DelBuono, P. Leruste and H. B. O'Connell, Eur. Phys. J. C 31 (2003) 525 arXiv:nucl-th/0306078.

[6] F. Ambrosino et al. [KLOE collaboration], Phys. Lett. B 669 (2008) 223 arXiv:0807.4909 [hep-ex]].

[7] Y. S. Tsai, Phys. Rev. D 4, 2821 (1971) [Erratum-ibid. D 13, 771 (1976)].

[8] M. Davier et al., arXiv:0906.5443 [hep-ph].

[9] C. E. Wolfe and K. Maltman, arXiv:0908.2391 [hep-ph].

[10] R. R. Akhmetshin et al. [CMD-2 Collaboration], Phys. Lett. B 527 (2002) 161 arXiv:hep-ex/0112031; Phys. Lett. B 578 (2004) 285 arXiv:hep-ex/0308008.

[11] R. R. Akhmetshin et al. [CMD-2 Collaboration], Phys. Lett. B 648 (2007) 28 arXiv:hep-ex/0610021.

[12] G. J. Gounaris and J. J. Sakurai, Phys. Rev. Lett. 21 (1968) 24.

[13] C. P. Shen et al. [Belle Collaboration], Phys. Rev. D 80 (2009) 031101 arXiv:0808.0006 [hep-ex]].

[14] B. Aubert et al. [BABAR Collaboration], Phys. Rev. D 74 (2006) 091103 arXiv:hep-ex/0610018]; Phys. Rev. D 76 (2007) 012008 [arXiv:0704.0630 [hep-ex]].

[15] C. P. Shen and C. Z. Yuan, arXiv:0911.1591 [hep-ex].

[16] B. Aubert et al. [BABAR Collaboration], Phys. Rev. Lett. 98 (2007) 212001 arXiv:hep-ex/0610057.

[17] Z. Q. Liu, X. S. Qin and C. Z. Yuan, Phys. Rev. D 78 (2008) 014032 arXiv:0805.3560 [hep-ex]].

[18] M. Ablikim et al. [BES Collaboration], Phys. Lett. B 645 (2007) 19 arXiv:hep-ex/0610023. 